\title{
Secolarizzazione, non secolarismo. II diritto nel nuovo rapporto tra pubblico e privato
}

\author{
Secularization, Not Secularism. The Law in the New \\ Relationship between Public and Private
}

\author{
Claudio SARTEA \\ Universitá degli Studi di Roma «Tor Vergata» (Italia) \\ claudio.sartea@uniromaz.it
}

\begin{abstract}
Riassunto: L'articolo cerca di approfondire il significato di termini come secolarizzazione e secolarismo, anche per distinguere tra un atteggiamento ideologico pregiudiziale nei riguardi del dialogo tra religione e sfera pubblica, ed attuali orientamenti aperti all'interazione tra discorso religioso e discorso pubblico come riflesso della continuità tra sfera privata e sfera pubblica. Tutto ciò ha molto da dire per la riflessione sul diritto, sul fondamento dei diritti e sulla stessa storia della civiltà giuridica, soprattutto di quella occidentale: per la quale il ruolo svolto in particolare dalla religione cristiana si rivela essenziale per la comprensione di principi come quello di uguaglianza e di laicità, nonché per la fondazione dei diritti umani.
\end{abstract}

Parole chiave: secolarismo; laicità; diritto; uguaglianza; diritti umani; religione; sfera pubblica; pubblico e privato.

\begin{abstract}
The article seeks to deepen the meaning of terms such as secularization and secularism, also to distinguish between an ideological attitude regarding dialogue between religion and the public sphere, and the current open guidelines for interaction between religious discourse and public discourse as a reflection of continuity between the private sphere and the public sphere. All this has a lot to say on the thought about law, about the foundation of the rights and about the history of juridical civilization, especially in the western culture: for which the role played by the Christian religion in particular is essential for the understanding of principles such as equality and secularity as well as the foundation of human rights.
\end{abstract}

Keywords: secularization; secularism; secularity; law; equality; human rights; religion; public space; public and private.

\section{PREMESSA}

$\square$

ul piano terminologico, occorre premettere una distinzione che considero essenziale sia dal punto di vista analitico che dal punto di vista euristico: quella tra «secolarizzazione» e «secolarismo». Nel lessico che adotto qui, la prima è un processo storico e culturale tipicamente occidentale e certamente ispirato dal vangelo cristiano. Il secondo, invece, è sostanzialmente una ideologia $\mathrm{o}$, se si preferisce, una maniera di guardare alla realtà (ed in particolare, per quel che soprattutto qui interessa, una maniera di guardare alla distinzione tra pubblico e privato in materia religiosa): quello che in lingua castigliana viene con efficace espressione definito «cosmovisión»e, nella 
lingua di John Rawls, «comprehensive doctrine». Cercherò di spiegare meglio qui di seguito questa distinzione, che, lo ripeto, considero molto rilevante.

È certamente nelle corde specifiche dell'annuncio cristiano (ancor prima della sua organizzazione teologica consapevole, che comincia con la patristica attorno al terzo secolo e giunge a maturità con Tommaso d'Aquino), per così dire sta nel cuore del kérygma caratteristico già dell'Antico Testamento ma poi in maniera più marcata e radicale (e vedremo subito perché) con il Vangelo cristiano, la valorizzazione positiva della realtà - cioè del saeculum, che appunto è termine semanticamente ricco per indicare lo spazio ed il tempo presenti, la realtà nella sua consistenza empirica e nel dinamismo della storia: forse, la traduzione concettualmente più precisa di questo complesso termine latino è la parola italiana «mondo».

Il testo sacro ebraico e cristiano esordisce ${ }^{1}$, come tutti sanno, con la celebrazione della prima opera di Yahweh, che è proprio la creazione del mondo: del resto, la stessa possibilità di descrivere azioni esige il tempo, ed il tempo esige la materia: ma se Dio è spirito (così lo considerano senza esitazione e fin dall'inizio gli Ebrei, e questo è un consapevole contrassegno identitario del Popolo eletto in dialettica con tutti gli altri popoli e le loro fedi o plurali - politeismo - o materialistiche o comunque non trascendenti - antropomorfismo, naturalismo, immanentismo, panteismo, etc.-), la prima opera divina di cui si può parlare in qualunque testo sacro non può che essere appunto la creazione del mondo, l'inizio dell'esistenza della materia. Ora, il fatto che la si celebri e non la si consideri - come pure è stato fatto: sia prima o al di fuori del cristianesimo con le dottrine emanazionistiche (per esempio di un Plotino), sia dopo il cristianesimo con la Gnosi, che correttamente è stata contestata dai primi teologi cristiani (Ireneo di Lione ${ }^{2}$ ) come ritorno al passato, al paganesimo - come un decadimento del divino, una sua corruzione, la sconfitta del Dio buono ad opera di un impersonale principio maligno o di un misterioso Dio cattivo -, è già sintomatico di quella visione positiva della materia, del corpo, della carne, che nonostante alcuni passi della stessa rivelazione vetero prima e neotestamentaria poi resiste in tutto il depositum fidei cristiano. Che culmina come dicevamo nel Vangelo: esso non è anzitutto una narrazione o una storia,

1 Il riferimento è ovviamente al primo libro della Bibbia, la Genesi, ed in particolare ai capitoli primo (tradizione iahvista), e secondo (tradizione elohista).

2 IRENEO Di LiONE, Adversus Haereses: innumerevoli i passi del suo capolavoro in cui presenta la critica della gnosi neopagana ed afferma con vigore e bellezza l'autentica novità cristiana: per tutti si veda lo splendido IV, 34, 1. 
ma l'annuncio di un mistero o se si vuole di un dogma rivoluzionario, quello della Incarnazione di Dio in corpo umano, che equivale all'ingresso dell'assoluto nel contingente, dell'eterno nel tempo, dello spirito che aveva creato $e x$ nibilo dentro la sua stessa creazione, appunto il mondo, il saeculum. Al centro del cristianesimo c'è dunque, inamovibile, una rivelazione destinata a rimanere esclusiva ed unica nell'esperienza religiosa dell'umanità: la secolarizzazione di $\mathrm{Dio}^{3}$.

Sia che ci si muova dall'interno del cristianesimo stesso, sia che si guardi a tutto questo dal di fuori di un'adesione religiosa e ci si voglia limitare all'analisi dei processi culturali, prendere sul serio detta «secolarizzazione di Dio» implica la massima valorizzazione possibile del secolo: dell'umano, dello storico, del materiale, dell'empirico, del contingente, persino del quotidiano, dell'esperito, del corporeo. Si tratta di un criterio fondamentale del cristianesimo, sebbene abbia avuto altalene di luce ed ombra nell'elaborazione storica della sua dottrina.

In ogni caso, ai giorni nostri è stato messo in primo piano dalla dottrina teologica proclamata dal Concilio Vaticano II (1959-1965): basti leggerne la Costituzione considerata da molti come la più originale e feconda, Gaudium et Spes, per rendersene conto; in particolare, nel cruciale capitolo 22 ove si incontrano le celebri espressioni che seguono: «Solamente nel mistero del Verbo incarnato trova vera luce il mistero dell'uomo. Adamo, infatti, il primo uomo, era figura di quello futuro $(\operatorname{Rm} 5,14)$ e cioè di Cristo Signore. Cristo, che è il nuovo Adamo, proprio rivelando il mistero del Padre e del suo amore svela anche pienamente l'uomo a se stesso e gli manifesta la sua altissima vocazione. [...] Egli è 'l'immagine dell'invisibile Iddio' (Col 1, 15), è l'uomo perfetto che ha restituito ai figli di Adamo la somiglianza con Dio, resa deforme già subito agli inizi a causa del peccato. Poiché in lui la natura umana è stata assunta, senza per questo venire annientata, per ciò stesso essa è stata

3 Con la profondità che gli è propria, Ratzinger evidenzia che la cristologia (la branca della teologia cristiana che ha meditato riflessivamente il mistero di cui stiamo parlando, certamente essenziale per il cristianesimo) si è storicamente prodotta in due filoni principali di ricerca: quello appunto dell'incarnazione e quello della croce. Il primo, come osserva questo autore, che fissa lo sguardo ontologicamente sull'essere di questo Dio incarnato, il secondo (più antico e corrispondente alla genuina proposta paolina, ripresa anche dalla tradizione protestante) sull'agire di Dio, sull'avvenimento della redenzione attraverso il sacrificio: RATZINGER, J., Introduzione al Cristianesimo, Queriniana, Brescia, 2005, p. 219. È evidente che qui adotto specialmente la prima prospettiva, che tra l'altro mi sembra più radicale (agere sequitur esse, come vuole il vetusto brocardo). 
anche in noi innalzata a una dignità sublime. Con l'incarnazione il Figlio di Dio si è unito in certo modo ad ogni uomo. Ha lavorato con mani d'uomo, ba pensato con intelligenza d'uomo, ha agito con volontà d'uomo, ba amato con cuore d'uomo. Nascendo da Maria vergine, egli si è fatto veramente uno di noi, in tutto simile a noi fuorché il peccato. [...] Soffrendo per noi non ci ha dato semplicemente l'esempio perché seguiamo le sue orme ma ci ha anche aperta la strada: se la seguiamo, la vita e la morte vengono santificate e acquistano nuovo significato. Il cristiano poi, reso conforme all'immagine del Figlio che è il primogenito tra molti fratelli, riceve 'le primizie dello Spirito' $(\mathrm{Rm} 8,23)$ per cui diventa capace di adempiere la legge nuova dell'amore». È impossibile sminuire la portata di questo testo, che del resto è stato al centro del magistero di Giovanni Paolo II (autentico araldo mondiale della dottrina conciliare), e dei suoi successori.

Sul piano politico e, per quel che qui ci interessa specificamente, giuridico, questa rivoluzione culturale ha avuto il suo coerente sviluppo fin dal celeberrimo dictum evangelico che fonda la dottrina cristiana della laicità: «Reddite quae sunt Caesaris Caesari, et quae sunt Dei Deo» ${ }^{4}$. Non è casuale il parallelismo di questa esortazione - che distingue senza separare le due istanze temporale e spirituale dell'autorità e se si vuole del potere - con la stessa struttura della personalità di Cristo che la enuncia, «perfetto uomo e perfetto Dio» ${ }^{5}$ : completamente creato in quanto uomo, e quindi da rispettare appieno nella sua umanità, e completamente increato in quanto Dio, e quindi da adorare nella sua divinità. L'autonomia delle due sfere - che, lo ripeto, non implica la separazione, proprio come nell'unione ipostatica non vi è lacerazione o schizofrenia ma appunto unione, armonia, equilibrio e cooperazione - riflette misteriosamente la stessa persona di Cristo. Era insomma non solo inevitabile, ma doveroso che lo sviluppo della dottrina evangelica giungesse ad una compiuta secolarizzazione, che altro non è se non il rispetto e l'implementazione dell'indicazione matteana ${ }^{6}$ nei termini dell'autonomia e del rispetto - non certo della separazione né di una dialettica emancipazione -

4 Mt XXII, 21.

5 Secondo la formula divenuta canonica e quasi proverbiale, a cui ricorse per primo, in sede di definizione dogmatica, il Concilio di Nicea (325 d.C.), poi cristallizzatasi nella cosiddetta professio fidei niceno-constantinopolitana.

6 Peraltro non isolatamente: si veda anche il rifiuto di Gesù in ordine alla richiesta di dividere un'eredità tra due fratelli (Lc XII, 13-21), che contesta l'attribuzione (o, peggio, l'acquisizione) di poteri e funzioni temporali a chierici. 
della sfera temporale (scientifica ${ }^{7}$, etica, giuridica, politica, economica, e così via) da quella spirituale rivelata ${ }^{8}$.

Se la «secolarizzazione» riposa dunque al centro stesso della prospettiva cristiana, ed anzi sorprende che abbia impiegato tanto tempo - secoli, in un certo senso: o almeno, che nei secoli abbia spesso perduto la propria genuina forza e con essa buona parte della sua originalità'- ad affermarsi pienamente nell'autocomprensione cristiana, ben diverso è quello che abbiamo chiamato all'inizio di questa premessa «secolarismo». Come tutte le parole che terminano con questa desinenza, anche il secolar-ismo rappresenta in ultima analisi un'ideologia: una chiave di lettura unilaterale (e per questo, giocoforza, riduttiva) della realtà, compresa la realtà cristiana. L'importanza del «secolo» viene cioè radicalizzata fino al punto di considerarlo incompatibile con Dio: proprio quel Dio che il secolo lo ha creato e poi, secolarizzandosi, lo ha redento, viene dal secolarismo inaspettatamente e contraddittoriamente espulso dal secolo. Se per alcuni questa cacciata è semplicemente il segno dell'avvenuta maturazione del secolo stesso e dei suoi protagonisti, ormai letteralmente emancipati dal riferimento a Dio e quindi autori di un ostracismo che equivale al rovescio della cacciata dall'eden sofferta dai progenitori, per altri si tratta invece del definitivo superamento di una visione inautentica della realtà. L'a-teismo si fa così sistematico: ed è curioso che questo sia stato storicamente l'esito non dello scetticismo o del materialismo, ma dello spiritualismo più radicale, quello hegeliano. La difficile conservazione (tipicamente cristologica, e dunque inclusa in un mistero e mai

7 In tal senso il «disincantamento del mondo» (e segnatamente della natura) di cui parlò efficacemente Weber, M., La scienza come professione. La politica come professione (1919), trad. it. a cura di F. Tuccari, Mondadori, Milano, 2006, p. 46, intendendolo alla stregua di una delle cifre della modernità come impresa epistemologica, non è affatto estraneo alla religione cristiana, anzi ne è conseguenza diretta, proprio in opposizione consapevole (già implicita anche nella visione biblica ebraica) delle divinizzazioni del contingente prodotte dalle altre religioni positive. Ma va al di là della visione ebraica, ed in un certo senso rovescia quelle tradizioni e questa allo stesso tempo, l'idea cristiana secondo cui non è qualche creatura a divenire, agli occhi del credente stupefatto dalle sue spettacolari manifestazioni, qualcosa di divino, bensì è il divino a divenire creatura.

8 Per vari approfondimenti sia sul versante storico che su quello filosofico, si veda DonaTI, P. (a cura di), Laicità: la ricerca dell'universale nelle differenze, Il Mulino, Bologna, 2008. Dell'interessante e perspicua analisi storica e culturale effettuata in questo volume collettaneo da RHONHEIMER, M., Cristianesimo e laicità. Storia ed attualità di un rapporto complesso, pp. 27 e ss., esiste anche una versione in lingua castigliana: RHONHEIMER, M., Laicidad y Cristianismo, Rialp, Madrid, 2009.

9 Ne parla diffusamente, e ne tenta una spiegazione, RHONHeImer, M., op. cit., pp. 33 e ss. Per qualche considerazione ed indicazione in più sul faticoso percorso di questa dottrina, con specifico riferimento alla teologia del lavoro, sia permesso rimandare a SARTEA, C., Deontologia. Filosofia del lavoro professionale, Giappichelli, Torino, 2012. 
completamente comprensibile dalla nostra ragione) dell'equilibrio tra il polo divino e quello umano (e ricordiamo di nuovo che la celebre espressione descrittiva di Cristo e riassuntiva dell'unione ipostatica: perfectus Deus, perfectus homo, era la chiave del Credo niceno-costantinopolitano), minata dall'idea centrale della teologia hegeliana, quella di un progressivo mondanizzarsi dello spirito divino (che in realtà coincide con il divinizzarsi dell'umano e dello storico, con la metamorfosi del contingente nell'assoluto: fino al curioso sovrapporsi delle vicende prussiane di inizio Ottocento con il momento di definitiva autorivelazione ed autocomprensione di Dio a se medesimo, che ovviamente trova pienezza nella mente di Hegel, il pensatore che infine lo ha teorizzato), si trasmuta (è proprio il caso di ricorrere al lessico nietzscheano) nel suo esatto opposto, l'ateismo sistematico delle ideologie tardo-ottocentesche e poi novecentesche. Superati i tentativi di implementazione sociopolitica dei nazionalismo e dei comunismi più brutalmente coerenti, ci ritroviamo ormai con una sola ideologia onnicomprensiva e tutto sommato universale: il secolarismo appunto, capace di assumere varie fogge in relazione ai fattori culturali condizionanti.

\section{SECOlarizZAZione, NON SECOlARISMO}

Il secolarismo tuttavia ha il grande difetto di fare torto alla realtà. La nota sentenza del Consiglio di Stato italiano sul mantenimento del crocifisso nelle aule d'istruzione pubblica obbligatoria ${ }^{10}$ - confermata almeno quanto al margine di apprezzamento dalla stessa Corte europea dei Diritti dell'Uomo di Strasburgo $^{11}-$, pur con tutte le critiche che le sono piovute addosso più o meno meritatamente ${ }^{12}$, ha ricordato tra le altre cose una verità piuttosto lapalissiana: e cioè che il fenomeno religioso è un fatto collettivo, con manifestazioni storiche così generalizzate da far considerare l'ateismo (e dunque anche la pretesa secolarista) più come un'eccezione che come la regola antropologica ${ }^{13}$.

10 Decisione del Consiglio di Stato italiano, 13 gennaio-13 febbraio 2006, n. 556.

11 Sentenza della Corte Europea dei Diritti dell’Uomo del 18 marzo 2011, ricorso n. 30814/06, Lautsi e altri c. Italia (per la traduzione italiana di questa decisione si può consultare anche https://www.giustizia.it/giustizia/it/mg_1_20_1.page?facetNode_1=0_8_1_14\&contentId=SDU647659\&previsiousPage=mg_1_20) (30.08.2017).

12 Vedasi per esempio il faccia a faccia presentato dal numero 10 dell'11 marzo 2006 della rivista «Diritto e Giustizia».

13 Lo aveva notato persino de Tocqueville: «L'incrédulité est un accident; la foi seule est l'état permanent de l'humanité» (DE TOCQUeville, A., De la démocratie en Amérique, 1835, Flammarion, Parigi, 1981, I, p. 403). 
Qualsiasi cosa si pensi delle singole religioni, qualunque posizione s'intenda personalmente assumere circa il fatto religioso in sé e le sue connessioni con la vita etica dell'individuo, è innegabile che la cultura s'intesse ed alimenta del fattore religioso in modo estremamente rilevante ${ }^{14}$, a volte persino caratteristico, e non certo da oggi. La cosa non deve stupire, se si tiene conto del fattore collettivo del rito e del culto, che corrisponde alla domanda di sacro che proviene da tutti o quasi tutti i membri della collettività, a volte persino a prescindere dall'età e dal grado di alfabetizzazione. La storia sta lì a testimoniarlo in forme così chiare che non richiedono approfondimenti ulteriori in relazione a tutte le civiltà conosciute, così come è ben noto che la stessa possibilità di conoscerle deve molto, a volte tutto, al fenomeno religioso ed alle sue vestigia: il culto dei morti e l'erezione di templi costituiscono le principali fonti cognitive delle civiltà più remote, delle quali appunto solo ciò che era destinato ad oltrepassare il contingente è giunto fino a noi.

Se pertanto il secolarismo fa un torto all'antropologia culturale ed a quella filosofica, non possiamo considerarlo in modo diverso da un'ideologia. Con tutte le criticità che normalmente un'ideologia attira: convergenti nel difetto essenziale così ben messo in luce da Hannah Arendt, che definiva l'ideologia come «la logica di un'idea ${ }^{15}$, denunciandone in tal modo l'inevitabile tendenza a sacrificare parti di realtà sull'altare della convinzione e del fanatismo (sempre unilaterali e dunque riduttivi, perché se pur esiste la verità non è

14 Ricordiamo che questo punto è stato al centro del dialogo di Monaco tra Jürgen Habermas e Joseph Ratzinger, nel gennaio del 2004: un incontro talmente importante da meritare la pubblicazione su libro di entrambi i discorsi; si veda HABERMAS, J. e RATZINGER, J., Etica, religione e Stato liberale, Morcelliana, Brescia, 2005. Una perspicua lettura di quel dialogo in D'Agostino, F., Diritto e religione, Aracne, Roma, 2013. Oltre ai celebri discorsi del teologo poi eletto Pontefice romano (in particolare, per quel che qui ci interessa, i grandi discorsi giuridico-politici di Regensburg, New York, Parigi, Londra e Berlino, disponibili anche in internet ma raccolti e studiati criticamente in un prezioso volumetto a cura di CARTABIA M., e SimONCINI, A., La legge di re Salomone. Ragione e diritto nei discorsi di Benedetto XVI, Rizzoli, Milano, 2013), il seguito di quel dialogo sul versante habermasiano è compendiato nella raccolta di saggi HABERMAS, J., Tra scienza e fede (2005), trad. it. a cura di M. Carpitella, Laterza, Roma-Bari, 2006. Mi permetto anche di rinviare ad alcune riflessioni su quel dialogo e sui problemi ad esso sottesi proposte in SaRTea, C., Diritto secolare. Religione e sfera pubblica, oggi, Aracne, Roma, 2012, nonché in questa rivista SARTEA, C., «La laicidad de una razón pública ampliada», in Persona y Derecho, vol. 64 (2011), pp. 207-224.

15 ARENDT, H., Le origini del totalitarismo (1966), trad. it. a cura di A. Guadagnin, Ed. di Comunità, Milano, 1996, p. 642. Prediligo questa definizione, più semplice e al tempo stesso più profonda, perché più filosofica e radicale rispetto a quella, classica ma sociologica, proposta come noto da ManNHEIM, K., Ideologia e utopia (1953), trad. it. a cura di A. Santucci, Il Mulino, Bologna, 1957. 
pienamente conoscibile da una singola mente onnicomprensiva $\left.{ }^{16}\right)$ : $\ll$ Le ideologie ritengono che una sola idea basti a spiegare ogni cosa nello svolgimento dalla premessa, e che nessuna esperienza possa insegnare alcunché dato che tutto è compreso in questo processo coerente di deduzione logica. Il pericolo inerente al passaggio dall'inevitabile insicurezza del pensiero filosofico ${ }^{17}$ alla spiegazione totale di un'ideologia e della sua Weltanschauung non consiste tanto nel lasciarsi irretire da un'ipotesi spesso volgare, ma sempre acritica, quanto nell'abbandonare la libertà implicita nella capacità di pensare per la camicia di forza della logica, mediante la quale l'uomo può farsi violenza quasi con la stessa brutalità usata da una forza esterna $\gg^{18}$. Se vogliamo mantenerci liberi di pensare, più che mai ora che constatiamo - come fanno da tempo i sociologi della religion $\mathrm{e}^{19} \mathrm{ma}$ anche i filosofi teoretici meno condizionati dai tabù culturali tardoilluministici ${ }^{20}$ - il riemergere massiccio del fenomeno delle credenze in tutti gli strati sociali ed in tanti Paesi e culture che sembravano essersene definitivamente congedati, è insomma necessario andare oltre il secolarismo, anche per risemantizzare una relazione - essenziale per la riflessione giuridica e politica che deve stare specialmente a cuore ai cultori delle scienze sociali come quella tra pubblico e privato.

È stato ben scritto che «la modernità pensa Dio o come sopravvivenza superstiziosa o come luce di una ragione immanente al mondo e alla sua storia. La novità è che questi due modi di pensare oggi cadono. Che la religione non sia una superstizione lo si vede dal fatto che, proprio quando tutti i miti vengono abbattuti, il bisogno di una realtà soprannaturale, di un Essere Altro che non può essere racchiuso in nessun luogo e in nessun mito, non scompare, ma anzi si fa più forte. Che non sia la luce di una qualche ragione immanente alla storia lo si vede dal fatto che il mondo perde non solo la fede nella ragione, ma la stessa ragione $\gg^{21}$.

16 Come ha profondamente notato già Gadamer, è la fusione di orizzonti il massimo risultato dell'euristica non ideologica: GADAMER, H., Verità e metodo (1960), trad. it. a cura di G. Vattimo, Bompiani, Milano, 2000.

17 Che non ne è certo il difetto: v. sul punto D’Agostino, F., Corso breve di filosofia del diritto, Giappichelli, Torino, 2011, p. 18.

18 ARENDT, H., op. cit., p. 644.

19 Basti menzionare gli studi di J. CASANOVA: una sintesi molto chiara in «Rethinking Secularization: A Global Comparative Perspective», in The Hedgehog Review, Springer \& Summer 2006, pp. 7ss.

20 Coraggioso in tal senso il tentativo di AUDI, R., La razionalità della religione (2011), trad. it. a cura di A. Lavazza, Raffaello Cortina Editore, Milano, 2014.

21 Donati, P., La matrice teologica della società, Rubbettino, Soveria Mannelli, 2010, p. 47. 


\section{FEDE PERSONALE, RELIGIONE COLLETTIVA: UN LABIRINTO EVOLUTIVO}

Abbiamo visto che possiamo dare per scontato, e non bisognoso di ulteriore dimostrazione e messa in discussione, il fatto che il fenomeno religioso sia praticamente universale nel tempo e nello spazio ${ }^{22}$ : anche in tempi come $i$ nostri, in cui alcune aree del mondo sono certamente influenzate dalla pretesa secolarista, si è da più voci parlato di «ritorno del sacro» ${ }^{23}$, di «nuova evangelizzazione $»^{24}$, di «post-secolarismo» ${ }^{25}$. Il successo del secolarismo come visione del mondo è durato dunque l'espace d'un matin, persino nelle fronde più intellettuali e culturalmente sofisticate delle nostre società avanzate. L'illuminismo è stato superato molto più velocemente dei processi culturali che lo hanno preceduto: il tentativo di dargli un significato permanente (una sorta di eternizzazione dell'età adulta del criticismo verso le convenzioni, le tradizioni irriflesse, e così via), si è rivelato appunto poco più di questo, un tentativo, anzi, peggio, una paradossale mummificazione.

Qualcosa certamente è cambiato, e tutti, anche le personalità religiose, lo considerano un punto di non ritorno e persino «un duplice compito: spoliticizzare la religione e de-sacralizzare la politica $\gg^{26}$. Ma questo, come opportunamente ha chiarito Innerarity, non significa affatto che la religione scompaia o debba scomparire dall'orizzonte e dal discorso pubblico: «Quanto stiamo dicendo esige la messa a fuoco della differenza tra sfera privata e sfera pubblica, che per quanto mobile e complessa non ha perso affatto la sua rilevanza nemmeno per quanto riguarda la questione religiosa. Il fatto che le religioni

22 Possimo ancora ricordare Tocqueville, che lo dice in una forma particolarmente bella: «Seul entre tous les êtres, l'homme montre un dégoût naturel pour l'existence et un désir immense d'exister: il méprise la vie et craint le néant. Ces différents instincts poussent sans cesse son âme vers la contemplation d'un autre monde, et c'est la religion qui l'y conduit. La religion n'est donc qu'une forme particulière de l'espérance, et elle est aussi naturelle au coeur humain que l'espérance elle-même. C'est par une espèce d'aberration de l'intelligence, et à l'aide d'une sorte de violence morale exercée sur leur propre nature, que les hommes s'éloignent des croyances religieuses; une pente invincible l'y ramène». (DE TOCQUEVILle, A., De la démocratie en Amérique, cit., I, p. 403).

23 Per esempio sulle colonne del prestigioso The Wall Street fournal, Higgins, A., «In Europe, God is (not) dead», July 14, 2007.

24 Secondo l'espressione coniata da GIOvanNi PAOLO II sin dal 1985, e poi ripresa dai suoi successori.

25 Habermas, J., Tra scienza e fede, cit.

26 InNeraritY, D., Il nuovo spazio pubblico (2006), trad. it a cura di M. Mellino, Meltemi, Roma, 2008, p 44. 
si siano privatizzate significa che esse costituiscono oggi soltanto una parte dello spazio pubblico, che restano comunque qualcosa di diverso dal principio dell'autorità pubblica [...]. Le credenze sono elementi legittimi della società civile e possono chiaramente intervenire nel dibattito pubblico con la stessa legittimità di qualunque altra convinzione politica o morale. Ma proprio questo va ribadito: con la stessa legittimità e allo stesso titolo» ${ }^{27}$. Tra l'altro, questo processo consegna l'esperienza religiosa ad un'adesione più profonda da parte delle singole coscienze, e quindi in qualche modo la fa maturare, rendendola più consapevole. $\mathrm{O}$ almeno, come è stato detto con sintesi efficace ${ }^{28}$, procediamo tra la tendenza verso il believing without belonging ${ }^{29}$ e quella verso il belonging without believing ${ }^{30}$ : il che in fondo ribadisce da una nuova prospettiva la natura labirintica, e non monodirezionale né progressiva, dell'andamento di questi complessi processi ${ }^{31}$.

Uno dei maggiori sociologi contemporanei, scomparso di recente, Peter Berger, che fu tra i primi ad annunciare il secolarismo (nel senso da me indicato sopra: nel suo lessico troviamo la parola «secolarizzazione», di cui però abbiamo visto la più complessa semantica), come un processo culturale evolutivamente inevitabile e soprattutto irreversibile, da anni ha riconosciuto e ripetuto che siamo già entrati in una fase di recupero o riscoperta del sacro $^{32}$, spesso limitandosi con onestà sociologica ad annotare fenomeni che nessuno può mettere in discussione, e senza la necessità di evocare quelle manifestazioni di ritorno del sacro (sempre che lo siano davvero, che siano cioè autentiche: cosa che molti negano, anche tra le autorità religiose) che producono fanatismo e violenza persino attraverso forme organizzate di terrorismo internazionale: del resto, è piuttosto evidente che connotare in tal senso il ritorno del sacro si espone deplorevolmente alla critica proprio di quei laicisti ad oltranza i quali attribuiscono alle religioni positive la colpa di ogni male sociale, con un cortocircuito logico inaccettabile

7 INNERARITY, D., op. cit., p. 45.

28 Casanova, J., op. cit., p. 14.

29 DaVIE, G., Religion in Britain since 1945: Believing Without Belonging, Blackwell, Oxford, 1994.

30 HeRvieu-LÉger, D., «Religion uns Sozialer Zusammenhalt in Europa», en Transit: Europäische Revue, 26 (Summer 2004), pp. 101-119.

31 Forse è per questo che le posizioni unilaterali risultano sempre semplificatorie o persino grossolane, anche quando provengono da studiosi del rango di ENGELHARDT jr., H.T., Dopo Dio. Morale e bioetica in un mondo laico (2014), trad. it. a cura di R. Rini, Claudiana, Torino, 2014.

32 Si pensi anche soltanto allo studio intitolato A Far Glory: The Quest for Faith in an Age of Credulity, del 1992, ed alla raccolta da lui curata nel 1999, The Desecularization of the World: Resurgent Religion and World Politics. 
sul piano storico e sociale, e tanto più grave perché oblitera il ruolo decisivo delle religioni nel progresso e nella fioritura umana individuale e collettiva ${ }^{33}$. Un caso singolare è quello di Martha Nussbaum, che pur accodandosi al corteo dei polemisti ${ }^{34}$, secondo i quali è proprio la religione con le sue pretese di certezze e persino di verità assolute ad alimentare l'intolleranza e presto o tardi a volgersi in violenza contro gli altri, chiunque essi siano, colpevoli semplicemente di non aderire alla verità che per definizione è unica; pure già da qualche anno, riflettendo sul ruolo imprescindibile che la retorica, l'arte, la musica hanno nella generazione delle «emozioni politiche» ${ }^{35}$ che costituiscono la linfa della vitalità democratica, non evidenzia con la forza sufficiente che i suoi campioni prediletti (Martin Luther King, Gandhi, Tagore) sono spesso personalità profondamente e dichiaratamente religiose. Al contrario, preferisce far precedere l'analisi del loro contributo storico ed ideale da una poco originale riflessione sull'illuministico (ed un po' trito) concetto di «religione dell'umanità» (Rousseau, Comte, Stuart Mill): con il che peraltro non fa che confermare l'impressione che il fatto religioso, comunque si configuri, sia indispensabile generatore di vitalità etica $\mathrm{e}$ politica.

Gli esempi portati da Berger sono molto più diffusi, generalizzati ed elementari, oltre che pacifici ed a volte persino entusiasmanti, come le Giornate Mondiali della Gioventù di Giovanni Paolo II, la diffusione del cattolicesimo in contesti vincolati a tradizioni religiose e culturali antichissime e da sempre estranee ad esso, come quelle orientali (e per converso la strenua resistenza di posizioni scettiche o antireligiose nella vita ed ancor di più nell'accademia europea), la sopravvivenza del religioso nel tessuto socioculturale statunitense, caratteristica di un'idea di laicità molto lontana dal laicismo europeo di marca francese ${ }^{36}$, e così via.

Un contributo anche più robusto, sotto il profilo filosofico e della storia delle idee, alla riflessione sugli andirivieni labirintici e tutt'altro che lineari

33 La più decisa apologia dell'apporto cristiano al progresso è probabilmente ai nostri giorni l'opera del sociologo americano Rodney STARK: si veda in particolare La vittoria della ragione. Come il Cristianesimo ha prodotto libertò, progresso e ricchezza (205), trad. it. a cura di G. Tonoli, Lindau, Torino, 2006.

34 Nussbaum, M., «Religious Intolerance», in Foreign Policy (October 23, 2009).

35 Nussbaum, M., Emozioni politiche. Perché l'amore conta per la giustizia (2013), trad. it. a cura di R. Falcioni, Il Mulino, Bologna, 2014.

36 Alcuni importanti approfondimenti sulla differenza tra i due modelli (che di per sé è un topos della letteratura sulla laicità e la secolarizzazione), in DONATI, P. (a cura di), Laicità: la ricerca dell'universale nelle differenze, cit., nonché $\mathrm{P}$. Donati, La matrice teologica della società, cit. 
dei rapporti tra fedi personali, religioni collettive e dimensione pubblica (e quindi anche giuridica) dell'esistenza delle persone, è quello proveniente dalla ricognizione genealogica di un profondo conoscitore della civiltà occidentale, Charles Taylor, che dieci anni fa pubblicò uno studio monumentale $^{37}$ su quella che lui chiamò «l'età secolare ${ }^{38}$, cercando di rintracciarne le matrici spirituali profonde, gli sviluppi successivi, e gli orizzonti verso cui attualmente sembra orientarsi. $\grave{E}$ anche alla luce della sua ermeneutica, che si può condividere o meno ma certamente non manca di acume e sensibilità critica, che di seguito tento conclusivamente di ricavare alcune indicazioni spero utili per proseguire e approfondire la riflessione sul rapporto tra pubblico e privato nel postsecolarismo, e quindi sullo spazio, la funzione e l'idea stessa del diritto e dei diritti in relazione alle fedi nel nuovo, cangiante contesto socioculturale.

\section{PubBlico E PRIVATO IN «EPOCA SECOLARE»: \\ QUEL CHE IL DIRITTO DEVE ALLA RELIGIONE}

Un primo aspetto che considero fondamentale per risemantizzare oggigiorno la relazione tra pubblico e privato, è quello della nuova cultura dell'uguaglianza. L'uguaglianza, che già per Aristotele era la chiave di volta ed il senso della giustizia (tutti ricorderanno che secondo lo Stagirita ogni virtù rientra nella giustizia generale come rispetto delle leggi, ma la virtù della giustizia in particolare altro non è che rispetto dell'uguaglianza ${ }^{39}$ ), solo modernamente ha avuto però la forza socioculturale di sfondare le barriere ideologiche della selezione sociale e sessuale, e non sempre né dovunque con la stessa forza ed effettività. Difficile però negare che sul piano dell'acquisizione teorica quello dell'uguaglianza sia divenuto un principio generale a partire dalla rivelazione cristiana ${ }^{40}$ :

37 Definito da Robert BELLAH «one of the most important books to be written in my lifetime» https://tif.ssrc.org/2007/10/19/secularism-of-a-new-kind (04.09.17).

38 TAYLOR, C., L'età secolare (2007), trad. it. a cura di P. Costa, Feltrinelli, Milano, 2009.

39 ARISTOTELE, Etica Nicomachea, V, 1130 b.

40 Il Vangelo è prodigo di indicazioni egualitarie, persino quando si tratta di difendere o criticare l'autorità ed il potere: per tutti cfr. Mt XXIII, 8. Nella predicazione paolina questa dimensione etica viene resa ancora più esplicita ed applicata a molteplici situazioni (con limiti e freni, ma certamente con forte carica innovativa): si veda per tutti Gal III, 28 («Non c’è più giudeo né 
un valore evangelico ${ }^{41}$, quindi, che tuttavia per la sua realizzazione pratica nelle istituzioni - tuttora ovviamente incompleta, ma assolutamente preponderante negli intenti e nelle dichiarazioni di principio - è stato oggetto di un lungo e complesso processo di riconoscimento ed implementazione, spesso in contrapposizione a consolidate assiologie discriminatorie prodotte da errate interpretazioni storiche persino del cristianesimo. Tutto ciò ha fatto sì che solo in epoca moderna si siano diffusi assetti democratici e nuovi equilibri circa il rapporto tra governanti e governati, tra base e vertice del potere, tra donne ed uomini, tra ricchi e poveri, tra abili e disabili, e così via. Sono ovviamente innumerevoli le ripercussioni operative, nella sfera giuridica, del progressivo sviluppo di questo principio, oggi unanimemente proclamato in tutte le Costituzioni democratiche ed in numerose Dichiarazione sovranazionali dei diritti. La ripercussione che qui merita specialmente di essere rimarcata riguarda il rapporto tra pubblico e privato, che chiede di essere ripensato non già né più in termini di potere (dominio e sottomissione), bensì in termini - anch'essi schiettamente evangelici ${ }^{42}$ - di servizio: il pubblico è la sfera in cui l'accesso è aperto (salve esigenze contestuali che inducano a selezionarlo, ma sempre in base a criteri di pari opportunità che impediscano discriminazioni arbitrarie - si pensi per esempio alla giustizia amministrativa come applicazione correttiva dei principi di buona amministrazione nella gestione della distribuzione dei beni e dell'accesso ai servizi pubblici); il privato è la sfera della libera concorrenza e della selettività bilaterale delle relazioni. Mi sembra che proprio da questa distinzione dipenda l'esistenza dello Stato sociale e della sua innervatura giuridica principale, i diritti sociali: che esistano questi diritti, e che vi siano soggetti, pubblici o privati delegati dal pubblico, tenuti alla loro soddisfazione, non può che essere conseguenza dell'af-

greco; non c'è più schiavo né libero; non c'è più uomo né donna, poiché tutti voi siete uno in Cristo Gesù»).

41 CотTA, S., «Né Giudeo né Greco, ovvero della possibilità dell'uguaglianza», in Rivista Internazionale di Filosofia del Diritto, 1976, pp. 331 e ss.

42 Lc XXII, 26. Persino un non teologo come DE ToCQUEVILLE, A., coglie finemente alcune manifestazioni sociali e politiche di questo principio nel cuore del cattolicesimo: «Je pense qu'on a tort de regarder la religion catholique comme un ennemi naturel de la démocratie. Parmi les différentes doctrines chrétiennes, le catholicisme me paraît au contraire l'une des plus favorables à l'égalité des conditions» (De la démocratie en Amérique, cit., I, p. 393). E poco oltre, in aperta polemica con i sostenitori del secolarismo europeo: «C'est le despotisme qui peut se passer de la foi, mais non la liberté. La religion est beaucoup plus nécessaire dans la république qu'ils préconisent, que dans la monarchie qu'ils attaquent, et dans les républiques démocratiques que dans toutes les autres» (p. 401). 
fermazione piena ed effettiva dell'uguaglianza tra tutte le persone ${ }^{43}$. Ecco per quale ragione la libertà positiva di ciascuno è chiamata a diventare un impegno sociale al pari della libertà negativa, come è stato vigorosamente ribadito dal premio Nobel per l'economia Amartya $\mathrm{Sen}^{44}$ con riferimento alle due libertà descritte da Isaiah Berlin ${ }^{45}$.

Un altro contributo innegabile della modernità come approdo della secolarizzazione, intesa nel senso cristiano sopra descritto, è dato dalla cultura dei diritti umani ${ }^{46}$, che ha condotto società ed istituzioni ad una nuova e doverosa sensibilità verso la persona umana, incompatibile con discriminazioni arbitrarie o peggio ideologiche (ed anche su questo fronte si nota che l'uguaglianza rimane elemento dominante di qualsiasi teoria giuridica). Sebbene le prime ratifiche ufficiali di questa dottrina (ancora peraltro in piena temperie nazionalistica, e dunque sotto il giogo di un condizionamento falsificante o per lo meno limitante), come il Bill of Rights su cui è costruito l'edificio democratico statunitense e la Dichiarazione dei diritti dell'uomo e del cittadino che animò la Rivoluzione Francese, fossero di chiara impronta acattolica o anticattolica (ma mai, ed anche questo va notato, del tutto areligiose ${ }^{47}$ ), la visione cattolica ufficiale dei diritti umani, dopo le iniziali e ben note perplessità ${ }^{48}$,

43 In Spaemann, che tra l'altro è un filosofo cristiano, questo principio diviene una specie di condizione metafisica sia per la precomprensione del fenomeno giuridico in quanto tale, sia per la sua stessa legittimazione: Spaemann, R., Persone. Sulla differenza tra 'qualcosa'e 'qualcuno' (1998), trad. it. a cura di L. Allodi, Laterza, Roma-Bari, 2005, p. 241.

44 SEN, A., La libertà individuale come impegno sociale (1990), trad. it. a cura di C. Scarpa, Laterza, Roma-Bari, 1997.

45 Berlin, I., Quattro saggi sulla libertà (1958), trad. it. a cura di M. Santambrogio, Feltrinelli, Milano, 1989.

46 Su cui si rimanda ad un recente numero monografico di questa stessa rivista per i necessari approfondimenti.

47 Come sostiene Donati, P., La matrice teologica della società, cit., p. 110, «nell'impostazione moderna, il potere politico deve immunizzarsi dalla religione, in quanto forza trascendente, e lo fa in due modi: (i) nel modello europeo, nato dalla rivoluzione francese e poi potentemente sintetizzato nella visione hegeliana della storia, la religione viene incorporata nello Spirito immanente che guida l'evoluzione della società: qui la religione appare come una delle forze soggettive e oggettive che debbono trovare il loro inveramento nello Stato; (ii) nel modello americano, nato nel nuovo mondo a partire dai Padri pellegrini, la religione viene considerata un fondamento autonomo della società, ma la si concepisce come ricerca della felicità individuale e quindi, per evitare che una concezione prevalga sulle altre, viene distaccata dal potere politico e dal raggio di azione dello Stato, separando nettamente le due sfere».

48 Per un'accurata ricostruzione della presenza della dottrina dei diritti umani nella Rivelazione e nel Magistero cattolici si veda ConcetTI, G. (a cura di), I diritti umani. Dottrina e prassi, AVE, Roma, 1982, specie pp. 21-243. 
da decenni li ha abbracciati senza riserve, ferma restando la necessità di una loro adeguata ed obiettiva fondazione ${ }^{49}$. Nessuno dubita che la diffusione del riconoscimento teorico e la generalizzazione dell'impegno volto alla protezione pratica dei diritti umani (spesso resi fondamentali con l'apposita enunciazione nelle Carte costituzionali oltre che nelle Dichiarazioni e Convenzioni elaborate in sede internazionale), abbia condotto ad una rilegittimazione del potere pubblico (nazionale e sovranazionale) in ordine proprio alla loro tutela: si è in altre parole compiuta una ridefinizione della sovranità in termini di «responsibility to protect $»^{50}$ e si è diffusa l'accettazione dell'idea secondo cui tanto di giustizia risiede nell'operato degli organi politici e giuridici quanto vi sia di rispetto e promozione dei diritti umani ${ }^{51}$. Questa stessa priorità logica ed operativa è anche plasticamente rappresentata dall'efficacia vincolante delle decisioni delle Corti sovranazionali come quella di Strasburgo per i Paesi europei e quella di San José de Costarica per i Paesi americani aderenti alle rispettive convenzioni di tutela dei diritti umani. In seno al nuovo modello di organizzazione giuridico-politica prodotto dalla teoria dei diritti umani si è poi sviluppato il pieno riconoscimento, almeno occidentale, della libertà religiosa come principio fondamentale, nel senso della radicale personalità della

49 È quel che, per quanto attiene al magistero cattolico, autorevolmente ribadisce il Compendio di dottrina sociale della Chiesa, per esempio al n. 152: «Il movimento verso l'identificazione e la proclamazione dei diritti dell'uomo è uno dei più rilevanti sforzi per rispondere efficacemente alle esigenze imprescindibili della dignità umana. La Chiesa coglie in tali diritti la straordinaria occasione che il nostro tempo offre affinché, mediante il loro affermarsi, la dignità umana sia più efficacemente riconosciuta e promossa universalmente quale caratteristica impressa da Dio Creatore sulla Sua creatura. Il Magistero della Chiesa non ha mancato di valutare positivamente la Dichiarazione universale dei diritti dell'uomo, proclamata dalle Nazioni Unite il 10 dicembre 1948, che Giovanni Paolo II ha definito 'una vera pietra miliare sulla via del progresso morale dell'umanità'». Tuttavia, come immediatamente dopo si legge, dev'essere chiaro che il loro fondamento ha un'origine trascendente, e proprio questa è la spiegazione ed al contempo la garanzia della loro intangibilità: «La fonte ultima dei diritti umani non si situa nella mera volontà degli esseri umani, nella realtà dello Stato, nei poteri pubblici, ma nell'uomo stesso e in Dio suo Creatore» (n. 153).

50 Come ricorda D'Agostino, F., Corso breve di filosofia del diritto, cit., p. 132. Sull'argomento, una preziosa monografia nella prospettiva giusfilosofica ci è offerta da FERnáNDEZ, E., De Vitoria a Libia: Reflexiones en torno a la responsabilidad de proteger, Comares, Granada, 2013.

51 Il recente congresso portoghese della Società Internazionale degli studiosi di filosofia giuridica e sociale ha messo a tema proprio la relazione tra pace (interna e globale) e (rispetto/promozione dei) diritti umani. L'intervento di Isabel TrujILLO, in particolar modo, ha messo in evidenza la progressiva ed ormai imprescindibile convergenza tra Stato di diritto e diritti umani: cfr. AA. VV., Peace based on human Rights, XXVIII World Congresso on the Philosophy of Law and social Philosophy, Lisboa, 2017. 
fede come esperienza, e quindi della sua incoercibilità: anzi storicamente è stata proprio la rivendicazione, la difesa e pian piano l'attribuzione di questo diritto fondamentale ad avviare e poi costantemente animare la battaglia per i diritti umani. È così che istanze e rivendicazioni provenienti dalla sfera privata assurgono immediatamente ad una significatività pubblica: e si apre il delicato dibattito sulla fondazione dei diritti dell'uomo ${ }^{52}$ in un'umanità che per alcuni è voluntas ut ratio e per altri è invece, assai diversamente, voluntas ut natura (se si vuole ricorrere allo schema dell'antropologia classica: ma si può anche citare l'aggiornamento di tale dualismo proposto da $\mathrm{Cotta}^{53}$, che preferisce parlare di dialettica ragione/pulsione): dibattito di cui qui è impossibile occuparsi espressamente ${ }^{54}$, ma che certo ha anche a che vedere con la visione che si intenda adottare in ordine all'origine dell'uomo ed al suo destino, cioè e per essenza con la visione religiosa che ciascuno - studioso, operatore giuridico o persona comune - ritenga di fare propria.

La laicità delle istituzioni pubbliche è un altro frutto che la modernità secolarizzata vanta come un proprio merito specifico: se non nella sua teorizzazione, certamente nella sua compiuta realizzazione ed ora nella sua accanita difesa. Ma tutti sanno che anche questo valore, o meglio il sistema assiologico che esso incornicia, così come l'uguaglianza e la persona quale fondamento dei diritti umani dipende in maniera piuttosto diretta dalla visione cristiana dell'uomo: fa parte in altri termini di quell'insieme di valori che secondo Böckenförde le società liberali presuppongono ma non saprebbero più difendere con buoni argomenti, giacché hanno escluso non solo la fede cristiana ma anche il pensiero metafisico ${ }^{55}$. Abbiamo già considerato la matrice evangelica (segnatamente, matteana) del principio di laicità, che rivoluzionò concettualmente gli assetti dei rapporti tra potere spirituale e temporale ${ }^{56}$ : sarebbero stati necessari secoli per

52 Da ultimo criticamente si veda Possenti, V., Diritti umani. L'età delle pretese, Rubbettino, Soveria Mannelli, 2017.

53 Cотта, S. Attualità e ambiguità dei diritti fondamentali, in Id., Il diritto come sistema di valori, San Paolo, Milano, 2004, p. 22.

54 Sia consentito rinviare a SARTEA, C., «Some Critical Remarks on Libertarian Natural Law», in Rivista di filosofia del diritto, VI, (2017), pp. 193-204.

55 BöCKENFÖRDE, E.-W., Diritto e secolarizzazione. Dallo Stato moderno all'Europa unita, a cura di G. Preterossi, Laterza, Roma-Bari, 2007, p. 53.

56 Oltre agli autori già menzionati si veda il prezioso Lessico della laicità, a cura di DALLA TORRE, G., Studium, Roma, 2007. In filosofia politica considera questo principio come l'origine teoretica di ogni separazione successivamente tentata anche WALZER, M., Sfere di giustizia (1983), trad. it. a cura di G Rigamonti, Laterza, Roma-Bari, 2008. 
trarne tutte le conseguenze operative, ma il dado era comunque tratto, e l'incantesimo del potere sacrale risultava per sempre messo in discussione ${ }^{57}$.

Connessa al principio di laicità rettamente inteso è anche, come abbiamo visto, la rivalutazione della secolarità come dimensione mondana: sotto le variegate forme della valorizzazione del mondo e della cultura, del corpo, del lavoro umano. Anche questo ha avuto e promette di continuare ad avere un forte impatto sugli ordinamenti giuridici, se si considera per esempio la crescente importanza dei diritti culturali da un lato, e della biogiuridica come protezione del corpo umano vivente dalle insidie del tecnomorfismo ${ }^{58}$, dall'altro; per non parlare del diritto al/del lavoro, centrale in tutti gli ordinamenti moderni, che l'hanno sviluppato potentemente anche se non sempre in piena coerenza con le sue premesse di giustizia, e che è entrato tra i principali temi della stessa dottrina sociale della Chiesa cattolica ${ }^{59}$.

Lo sviluppo di questo principio in ordine al rapporto tra pubblico e privato è pure piuttosto importante, perché conduce per mano al liberale riconoscimento di tutte le prese di posizione religiose - ivi incluse le loro manifestazioni pubbliche - con il solo limite dell'ordine pubblico (e del buon costume ove lo si consideri separabile da quello). Vi è naturalmente margine discrezionale per valutare quando ed in che misura tali limiti vengano varcati da una specifica manifestazione religiosa, individuale o collettiva ${ }^{60}:$ ma

57 Che si tratti di una scoperta cristiana, e segnatamente cattolica, è quel che D'Agostino profondamente osserva definendo sir Thomas More «patrono della laicità»: cfr. D'AGOSTINO, F., Jus quia justum. Lezioni di filosofia del diritto e della religione, Giappichelli, Torino, 2012, p. 16.

58 Jonas parla di un'etica per la civiltà tecnologica, stimolato da quella che lui stesso definisce «euristica della paura»: ma è chiaro che è proprio la sua sensibilità religiosa a guidare la sua affermazione centrale secondo cui «è bene che vi sia un futuro per l'umanità», da cui dipende tutto il suo sistema normativo postkantiano (Jonas, H., Il principio responsabilità. Un'etica per la civiltà tecnologica, 1979, trad. it. a cura di P.P. Portinaro, Einaudi, Torino, 2005).

59 Storicamente, la nascita di tale dottrina sociale si connette all'affermazione dei diritti dei lavoratori in un contesto sociale in cui erano oggetto di sistematico misconoscimento e violazione: LEONE XIII, Lettera Enciclica Rerum novarum, Roma, 1891.

$60 \mathrm{La}$ giurisprudenza degli ultimi anni, sia in Italia che altrove, è imponente: dal contenzioso sul conflitto tra diritto/dovere alla vita e alla salute e libertà religiosa dei testimoni di Geova in relazione alla necessità di trasfusioni di sangue (magari di minori, come nel celebre caso Oneda definito dalla Corte di Cassazione penale italiana nel 1983), o più recentemente la tensione tra esigenze di pubblica sicurezza e coltello rituale che completa l'abbigliamento dei sikh (Cassazione Penale, Sezione I, 15 maggio 2017, n. 24084), fino alle note decisioni sia nazionali che sovranazionali sull'ostensione di simboli religiosi in luoghi o nel contesto dell'esercizio di funzioni pubblici, e così via discorrendo fino all'episodio chiacchierato di questi giorni relativo all'affidamento di una minore cristiana ad una famiglia islamica che avrebbe costretto la bambina a pratiche musulmane implicanti l'abiura dalla fede di appartenenza. 
rimane acquisito il principio di laicità come criterio guida per evitare ogni discriminazione di principio, così come ogni privilegio socioculturalmente ingiustificato.

\section{CONCLUSIONI}

Le riflessioni proposte in queste pagine avevano essenzialmente lo scopo di fornire una cornice concettuale di riferimento utile ogniqualvolta si voglia mettere a tema l'odierno rapporto tra fede e diritto nelle società postsecolari. Che la fede sia un'istanza personalissima ed incoercibile, ospitata nell'area più intima e sacra della coscienza di ciascuno, è ormai elemento acquisito ed in via di principio fuori discussione, così come tutti coloro che guardano alla realtà senza paraocchi ideologici possono in essa riconoscere i segni permanenti del fenomeno religioso come avvenimento collettivo, luogo d'incontro e relazioni, dinamica cultuale, processo storico di massa in cui s'intrecciano novità e tradizioni, esperienze mistiche individuali ed eventi partecipativi. Sia il primo aspetto che il secondo esigono l'intervento dei diritti e del diritto come ordinamento: in ordine alla tutela di una libertà negativa, quella religiosa appunto, ed in ordine all'impegno ormai considerato proprio delle pubbliche istituzioni mirante al pieno sviluppo della persona in tutti i contesti che le sono caratteristici, ivi incluso quello spirituale. È tempo insomma di superare le angustie - anche normative - del secolarismo, e di prendere atto delle aspettative forti vantate da una secolarizzazione che lungi dal reprimere o limitare le manifestazioni della religiosità delle persone umane, è perfettamente in grado di riconoscerle, rispettarle, persino di valorizzarle, e sa leggere in esse l'umanità sia dei singoli sia delle loro relazioni sociali e di ogni successivo impegno per la casa comune.

Se, come alcuni ritengono con buone ragioni, il cancro profondo delle società contemporanee è l'individualismo ${ }^{61}$, che mina alla radice la possibilità stessa di una coesistenza che sia in quanto tale costruttiva, e produttiva in esclusiva di quei $\ll$ beni relazionali» ${ }^{62}$ che sono essenziali per la fioritura uma-

61 Interessante e ricco di spunti tra il filosofico ed il teologico il volume a cura di BREZZI F. e RUSSo, M.T., Oltre la società degli individui. Teoria ed etica del dono, Bollati Boringhieri, Torino, 2011.

62 Ne ha parlato per primo DONATI, P., Teoria relazionale della società, FrancoAngeli, Milano, 1991. 
na ${ }^{63}$, è difficile negare che la religione da sempre ha dato prova di una notevole attitudine a generare risorse di senso per la vita in comune, per il sacrificio, per la condivisione nella progettazione di un futuro collettivo ${ }^{64}$. In tal senso, la prospettiva religiosa ha, rispetto alla chiusura nell'immanenza propagandata dall'umanesimo esclusivo criticato da Charles Taylor ${ }^{65}$, oltre a diversi vantaggi operativi, un pregio fondamentale: si tratta infatti dell'unica motivazione all'azione (personale, collettiva, giuridica, politica, istituzionale), che non risulta mai di per sé manipolabile, definitivamente impossessabile o raggiungibile da chicchessia, ma sempre aperta come dialogo e come esigenza.

63 A parte l'immensa diffusione di questa prospettiva nella filosofia giuridica da Finnis in avanti, è utile qui ricordare che già Hart include varie sfaccettature della relazionalità umana (come risposta spontanea ed «ovvia» alla vulnerabilità individuale) tra quelli che lui chiama «truisms»: cfr. HART, H.L.A., Il concetto di diritto (1961), trad. it. a cura di M.A. Cattaneo, Einaudi, Torino, 2002.

64 Con atteggiamento aperto ed universalistico è quel che osserva anche il documento della Commissione Teologica Internazionale Alla ricerca di un'etica universale: nuovo sguardo sulla legge naturale, del 2009 (disponibile anche in internet: http://www.vatican.va/roman_curia/congregations/ cfaith/cti_documents/rc_con_cfaith_doc_20090520_legge-naturale_it.html).

65 TAYlor, C., L'età secolare, cit. 
0000 